\title{
Single particle wavefunction localizations in bulged CdSe nanowires
}

\author{
Zhengji Zhao ${ }^{1}$, Lin-Wang Wang ${ }^{1,1}$ and Fengmin $\mathrm{Wu}^{2}$ \\ ${ }^{1}$ Lawrence Berkeley National Laboratory, Berkeley, California 94720, U.S.A \\ ${ }^{2}$ Zhejiang Normal University, Jinhua, Zhejiang, 321004, P.R. China
}

Using atomistic empirical pseudopotentials, we have calculated the electronic structures of CdSe nanowires with a bulged area. The localized state wavefunctions and their binding energies are calculated, and their dependences on the bulged area shape are analyzed. We find that both the binding energy and the wavefunction localization strongly depend on the bulged area shape, with the most compact shape produces the largest binding energy and strongest wavefunction localization. We also find that the top of the valence band state has a weaker localization than the bottom of the conduction band state due to an effective mass anisotropy.

Keywords: nanowire, morphology, EPM, localization.

\footnotetext{
${ }^{1}$ Author to whom correspondence should be addressed: lwwang@lbl.gov
} 


\section{INTRODUCTION}

Nanostructure morphology often determines the electronic structures of the system. For example a change in the aspect ratio of the nanorod can induce a strong emission light polarization due to a state crossing in the valence band ${ }^{1}$, and different shapes of nanostructures, e.g, arrow shapes, tear drops and tetrapods ${ }^{2}$, can produce different electron hole localizations. As the synthesize techniques improve continuously, there are more and more control of the nanostructure shape and morphology. Thus it is of ultimate importance to study how the electronic structure changes when the nanostructure morphology changes.

In this paper, we study semiconductor nanowires. Nanowire can be synthesized by vapor-liquid-solid (VLS) method on a substrate ${ }^{3}$, or solution-liquid-solid (SLS) catalytic growth in a solvent ${ }^{4,5}$. It can also be synthesized by deposition along the surface steps ${ }^{6}$, and into the nanopores of aluminum oxide templates ${ }^{7}$. Besides, nanowires can be produced by attachment/aggregation of presynthesized nanodots and rods out of their dipole-dipole interactions ${ }^{8,9}$. Nanowires can be used in the future for electronic devices to replace the Si based technology, and can be used in solar cells and molecular sensors. In the electronic device and solar cell applications, the carrier conductivity in nanowire is one of its most important properties. While there are many factors that might affect the conductivity of a nanowire, for example the electron-phonon scattering, the surface roughness induced scattering, etc, here we study the effect of wavefunction localization which can be caused by a bulged area in a nanowire. Such localization can significantly change the carrier transport behavior of the nanowire since it interrupts the running wave 
Bloch state and can serve as a carrier stationary site. The understanding of such transport behavior needs to be started from the understanding of its electronic structures, e.g, the energy changes and wavefunction localizations, which will be studied here. Although current synthesize technique can produce extremely uniform nanowires, especially when the nanowire diameter is large (e.g, $>100 \mathrm{~nm}$ in the VLS method, and for the $>10 \mathrm{~nm}$ wires in the SLS synthesized wire), when the wire diameter decreases (e.g, around 2-4 $\mathrm{nm}$ ), and for the attachment/aggregation synthesized nanowires ${ }^{8}$, bulged areas are likely. After all, it is still a fundamental experimental challenge to synthesize uniform wires over long length for different semiconductor materials ${ }^{10}$. Thus there is of practical importance to study the effects of such bulged area. On the other hand, one can imagine to intentionally synthesize such bulged areas in the future (e.g, by attaching different sized nanorods using the aggregation process similar to that of Ref.8). This might be a useful way to control the nanowire electronic transport in the electronic devices.

We will use CdSe nanowires as the examples in the current study. As one knows, for an one dimensional system, any small potential well will produce a localized state. Thus, it is expected that any bulged area in an otherwise uniform and infinitely long nanowire will induce localized states. Obviously, for small perturbation cases where the majority of the localized single particle wavefunction is outside the bulged area, the larger the bulged area (the lump), the stronger the wavefunction localization and larger the corresponding eigenstate binding energy. Thus a more interesting topic will be to study how the shape of the bulged area (the lump) might change the localization and binding energy. To this aim, we have studied eight different systems with roughly the same lump size (the total extra $\mathrm{Cd}$ and $\mathrm{Se}$ atoms beyond the original uniform nanowire), but with 
different lump shapes. The lump shape ranges from a thin disk, to a long flat shell, and from a circular cross section, to elliptical and one sided cross section. We find that the shape can significantly alter the localization and binding energy, with the maximum localization and binding energy comes from the most compact shape of the bulged area cross section [10.5]. This is in contrast with the zeroth order perturbation theory under the simple effect mass theory. Under this theory, for small perturbations, the binding energy and localization should only depend on the total volume of the bulged area. We also find that the bulged area induces a stronger c-axis localization in the conduction band state than in the hole state, and the conduction band state wavefunction can more easily reach out to the bulged area.

\section{THE CALCULATION METHODS}

We will use the atomistic empirical pseudopotential method (EPM) to study the electronic structure of CdSe nanowire. This is a well established method which has produced electronic structures with excellent agreements with experiments for both CdSe nanowires $^{5}$ and quantum dots ${ }^{12}$. In the EPM method, the potential $\mathrm{V}(\mathrm{r})$ in the single electron Schrödinger's equation is described by the superposition of atomistic screened

potentials $\mathrm{v}_{\alpha}(r): V(r)=\sum_{R} \mathrm{v}_{\alpha}(r-R)$. The nanostructure is defined by a set of the atomic positions $\{\mathrm{R}\}$ for $\mathrm{Cd}$ and $\mathrm{Se}$ atoms within the wire/bulged area shape taken from their ideal bulk crystal structures. The atomic pseudopotential $v_{\alpha}(r)$ for atom type $\alpha$ is fitted to the experimental band structures, including the band gap, the special point energies, the effective masses, the deformation potentials, etc. In particular, for CdSe 
EPM, we have also fitted the EPM bulk electron wavefunctions to ab initio wavefunctions (with $99 \%$ overlap) ${ }^{13}$. Besides the local potential, there is also a nonlocal potential $\hat{V}_{N L}$, which is the same as the nonlocal part of the ab initio pseudopotential. The nonlocal potential part also includes the spin-orbit interaction, which is important to describe the CdSe top of valence band. In order to describe the spin-orbit interaction, the wavefunction $\psi(\mathrm{r})$ is described as a spinor with spin up and down components. Finally, the surface of the nanostructure is passivated with ligand potentials, which is fitted to remove the dangling bond states away from the band gap. The details of these potentials are described in Ref.12. After the potential V(r) for a given nanostructure is obtained, the single particle Schrödinger's equation

$$
\left[-\frac{1}{2} \nabla^{2}+V(r)+V_{N L}\right] \psi_{i}(r)=E_{i} \psi_{i}(r)
$$

will be solved by the folded spectrum method $(\mathrm{FSM})^{14}$. Here, the wavefunction is expanded by a planewave basis set, with an energy cutoff of 6.88 Ryd, and again it has the spin up and spin down components at any given spatial point $r$. The FSM only solves a few eigenstates near the edges of the band gap, thus it scales linearly to the size of the system. As a result, the computation of this approach scales linearly to the size of the system. This allows us to calculate a thousand atom system in a few hours on a parallel computer. In the current study, we will be focused on the wavefunction $\psi(\mathrm{r})$ and the corresponding eigen energies. We will show the results only for the conduction band minimum (CBM) state and valence band maximum (VBM) state since they have the strongest influence on the electronic transports. 
We have taken a $2 \mathrm{~nm}$ diameter wurtzite CdSe nanowire. This is close to the smallest nanowires synthesized experimentally ${ }^{5}$. The c-axis of the wire is in the (0001) direction of the wurtzite crystal structure. A bulged area is introduced by enlarging the diameter in one region. In the cross section, this enlarged area could be circular, elliptical, square, or attached from one side. In the cross section along the c-axis, the enlarged area could be a rectangle with different aspect ratios, a triangle, or a double-peak. In total, eight shapes are chosen to explore the variety of the morphologies. The extra number of $\mathrm{Cd}+\mathrm{Se}$ atoms (compared to the uniform nanowire) is about 250 for all these shapes. This amounts to increase the diameter of the wire by $60 \%$ in a length of 2 periods of the wurtzite unit cell in the c-axis $(14 \AA)$. In order to simulate an infinite nanowire, a periodic supercell with a c-axis length of $105 \AA$ ( 15 wurtzite unit cell periods in the z-direction) is used. This is much longer than the localized state decay length, thus the possible interactions between the periodic bulged areas are very small. In the other two directions, a $43 \AA$ length (10 periods of the wurtzite unit cell) equal sided parallelogram is used. The real space grid for this surpercell is $480 \times 180 \times 180$. The systems have about $1400 \mathrm{Cd}$ and Se atoms.

\section{THE RESULTS AND DISCUSSIONS}

The uniform nanowire has a VBM energy of $-4.912 \mathrm{eV}$ and CBM energy of $-2.349 \mathrm{eV}$, these should be compared with their bulk wurtzite counter parts of $-4.480 \mathrm{eV}$ and -2.683 $\mathrm{eV}$. The nanowire has a band gap of $2.563 \mathrm{eV}$, much larger than the bulk CdSe band gap of $1.797 \mathrm{eV}$, showing a strong quantum confinement effect for this small nanowire. The calculated nanowire band energies are in agreement with our previous calculated results for CdSe nanowire ${ }^{15}$. 
The eight bulged nanowires are listed in Table I, numerated from wire_1 to wire_8. Their shapes are described in cross sections along with the c-axis and perpendicular to c-axis. They all have roughly 250 extra $\mathrm{Cd}+\mathrm{Se}$ atoms. The shapes of all the bulged nanowires are shown in Fig.1 with orthogonal projections in both the side views and the top views. As we can see that, the shape ranges from tall in radial direction (wire_1), to medium (wire_2) and to thin (wire_3), and from circular cross sections (wire_1 to 5), to square (wire_6), elliptical (wire_7), and one-sided (wire_8) cross sections. Besides, for wire_4, the c-axis cross section is a triangle (or say an arrow), and for wire_5, it is consisted with two thin disks.

The eigen energies of these eight bulged nanowires are shown in Table I. They are listed as the binding energies $\triangle \mathrm{CBM}$ and $\triangle \mathrm{VBM}$ (the energy differences between the bulged nanowire $\mathrm{CBM} / \mathrm{VBM}$ and the uniform wire $\mathrm{CBM} / \mathrm{VBM}$, respectively). These binding energies range from $18 \mathrm{meV}$ to $97 \mathrm{meV}$. Thus, the shape can significantly changes the binding energy. Note that, if the bulged area is small, we can try to estimate its effects using effective mass perturbation theory. Here separating the Schrödinger's equation in cross section directions and c-axis direction, the cross section quantum confinement provides an effective quantum well potential for the c-axis wavefunction. This potential well is proportional to $1 / m^{*}(R+\Delta R)^{2}-1 / m^{*} R^{2} \approx 2 \Delta R / m^{*} R^{3}$, with $m^{*}$ being the effective mass, $R$ being the radius of the wire and $\Delta R$ being the increase of the radius due to the bulged area assuming a circular cross section. Then, using the zeroth order perturbation theory, the binding energy should be proportional to $\left.2 \Delta R L \psi\right|^{2} / m^{*} R^{3}$, 
where $\mathrm{L}$ is the bulged area length, and $\psi \mid$ is the wavefunction amplitude at the bulged area. If we assume that the bulged area only expands a small portion of the total wavefunction, then $\psi \mid$ depends on the wavefunction localization length, which in term depends on the binding energy itself. For small perturbation, $\Delta R L$ is proportional to the bulged area volume. Thus, according to this simple argument, for the same volume bulged area, the induced binding energy should be the same. This is apparently not the case we found. The big variation of the binding energy exists presumably because the simple effective mass theory is no longer valid for our small nanowire, and the bulged area is more than a perturbative change of the nanowire shape, which is certainly true for the thin disks like wire_5. It turns out that the triangle shaped bulged wire (wire_4) has the largest binding energy, presumably because it is the most compact structure (the increased radial size equals the c-axis size for the bulged area). On the other hand, the two-disk shape (wire_5) has the smallest binding energy, because the wavefunctions cannot take the advantage of the very thin disks.

Table I also shows that the binding energy of the VBM is very similar to that of CBM. This correlation between the VBM and CBM binding energies is shown in Fig.2 (a). As we can see from the fitted line, in average, VBM binding energy is about $90 \%$ of the CBM binding energy. This is a bit surprising because according to simple effective mass theory, the ratio of the binding energies should be inversely proportional to the ratio of the effective masses, and the CdSe VBM effective mass is larger than the CBM effective mass. Besides the reason that effective mass theory might not be valid for these small size systems, another possible reason is that the VBM energy is controlled by a mixture of heavy hole (in the cross section directions) and light hole (in the c-axis direction) 
effective masses. The average (to be more exact, the average in their inverses) of them is thus not too much larger than the CBM effective mass.

The CBM and VBM wavefunction square isosurfaces are shown in Fig.1 for all the 8 bulged nanowires. The same isosurface values of 0.0002 a.u is used for both the CBM and VBM wavefunction squares. The same wavefunctions are also shown in Fig.3, where instead of showing the isosurfaces, the wavefunction squares are first averaged in the cross sections, and then plotted in the c-axis directions in a logarithmic scale. This allows one to determine their exponential decay length. Assuming an exponential decay $\exp (-2 z / L)$ in the tail region of Fig.3, the wavefunction decay length $\mathrm{L}$ can be estimated. The results are listed in row 7 and 8 in Table I. From Fig.1, Fig.3 and Table I, we see that the VBM is in general less localized than the CBM. In the first glance, this might seem strange because one expects stronger localization of the VBM due to its larger effective mass for a same quantum well for VBM and CBM. This puzzle can be resolved by analyzing the details of VBM quantum confinement mechanism. First of all, a same bulged area doesn't mean it is the same effective z-direction (c-axis) quantum well potential for CBM and VBM. As discussed above, the effective potential well is proportional to $2 \Delta R / m^{*} R^{3}$. Thus larger the effective mass, smaller the effective potential well for the $\mathrm{z}$ direction Schrödinger's equation. As a result, the localization in $\mathrm{z}$ direction should be the same in the simple isotropic effective mass model regardless of the value of the effective mass. Another way to think about this is to look at the $3 \mathrm{D}$ effective mass Schrödinger's equation (with an isotropic effective mass). It is $-\frac{1}{m^{*}} \nabla^{2} \psi=E \psi$ inside the nanostructure, and $\psi=0$ outside the nanostructure. Thus the 
solution of $\psi$ doesn't depend on the value of effective mass $m^{*}$, it only depends on the shape of the nanostructure. While the CBM can be described by a single isotropic effective mass model, for the top of valence band Bloch state, it has an anisotropic effective mass. In the direction of the cross section ( $\mathrm{x}$ and $\mathrm{y}$ directions), it has a large heavy hole effective mass, while along the c-axis (z direction), it has a small light hole effective mass. Thus the heavy hole effective mass will create a relatively small effective potential well, and the small light hole effective mass in z-direction will make it difficult to confine the wavefunction with this potential well. This effective mass anisotropy of the VBM explains why it is less confined in the z-direction compared to the CBM state. The estimated exponential decay length L for the CBM and VBM are plotted versus the CBM and VBM binding energies in Fig.2 (b) and Fig.2 (c) respectively. As one can see, they roughly fall into the $1 / L=\alpha \sqrt{\Delta E}$ effective mass model relationship. The coefficient $\alpha=\sqrt{2 m^{* 1}}$, gives us the effective "effective mass $m^{*}$ " of these wavefunctions in the zdirection. These effectives masses from Fig.2 (b) and (c) are $0.18 m_{0}$ for CBM and 0.13 $m_{0}$ for VBM. They are similar to the bulk EPM effective masses of conduction band $\left(\sim 0.16 m_{0}\right)$ and light hole $\left(\sim 0.18 m_{0}\right)$. Finally, from the top view projections of Fig.1, we see that the CBM states extend more to the bulged area than the VBM states. This is also consistent with the fact that the CBM is more localized in the $\mathrm{z}$ direction in the bulged area.

\section{CONCLUSIONS}

We have calculated the CBM and VBM wavefunctions for eight bulged CdSe nanowires. We studied the shape dependences of the binding energies and wavefunction localizations of the localized CBM and VBM states in these bulged nanowires. We found 
that: (1) The binding energies depend strongly on the shape of the bulged area, with the most compact shape (the triangle one in wire_4) provides the largest binding energies. (2) There is a strong correlation of the VBM binding energies and CBM binding energies. In average, the VBM binding energy is about $90 \%$ of the CBM binding energy. (3) The VBM wavefunction is in general less localized in the c-axis. This is due to the anisotropy of the effective mass in the valence band state. (4) The wavefunction decay length in the tail region correlates with the binding energy according to the effective mass formula. (5) The CBM states are more spread out into the bulged area than the VBM states.

Acknowledgements: This wok was supported by the Director, Office of Science, Basic Energy Sciences, and Division of Material Science, of the U.S. Department of Energy under Contract No. DE-AC02-05CH11231. It used the resources of National Energy Research Scientific Computing Center (NERSC).

Table I. Binding energies and the wavefunction decay lengths of the eight bulged nanowires. The shape of the bulged area is described in the second and third rows of the table (see also Fig. 1) ("tall", "medium", "thin" describe the radial direction heights of the rectangles). The number of the atoms in the bulged area is listed in the row 4 . The binding energies of VBM and CBM, denoted as $\triangle \mathrm{VBM}$ and $\triangle \mathrm{CBM}$, are measured 
from their values in the uniform nanowire. The wavefunction decay lengths of VBM and CBM (L-VBM and L-CBM) are measured from the tail regions of the wavefunction in Fig.3.

\begin{tabular}{|c|c|c|c|c|}
\hline & Wire_1 & Wire_2 & Wire_3 & Wire_4 \\
\hline Cross-section (// $c$-axis) & Rect. (tall) & Rect. (medium) & Rect. (thin) & Triangle \\
\hline Cross-section $(\perp c$-axis $)$ & Circle & Circle & Circle & Circle \\
\hline \# of extra atoms & 243 & 267 & 246 & 255 \\
\hline$\Delta \mathrm{VBM}(\mathrm{meV})$ & 42 & 75 & 77 & 73 \\
\hline$\Delta \mathrm{CBM}(\mathrm{meV})$ & -49 & -72 & -78 & -97 \\
\hline L-VBM $(\AA)$ & 13.4 & 9.0 & 9.8 & 11.3 \\
\hline \multirow[t]{2}{*}{ L-CBM $(\AA)$} & 10.9 & 7.8 & 8.2 & 7.9 \\
\hline & Wire 5 & Wire 6 & Wire 7 & Wire 8 \\
\hline Cross-section (// c-axis) & Two-Peaks & Rectangle & Rectangle & Rectangle \\
\hline Cross-section $(\perp c$-axis $)$ & Circle & Square & Ellipse & One-sided \\
\hline \# of extra atoms & 228 & 261 & 243 & 249 \\
\hline$\Delta \mathrm{VBM}(\mathrm{meV})$ & 25 & 42 & 53 & 28 \\
\hline$\Delta \mathrm{CBM}(\mathrm{meV})$ & -18 & -52 & -49 & -19 \\
\hline L-VBM $(\AA)$ & 19.2 & 13.5 & 11.4 & 18.4 \\
\hline L-CBM $(\AA)$ & 17.7 & 10.4 & 12.3 & 19.1 \\
\hline
\end{tabular}


(1)
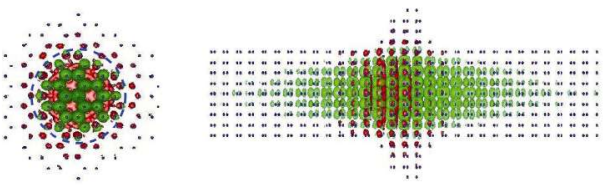

(2)
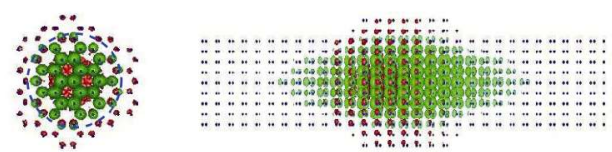

(3)
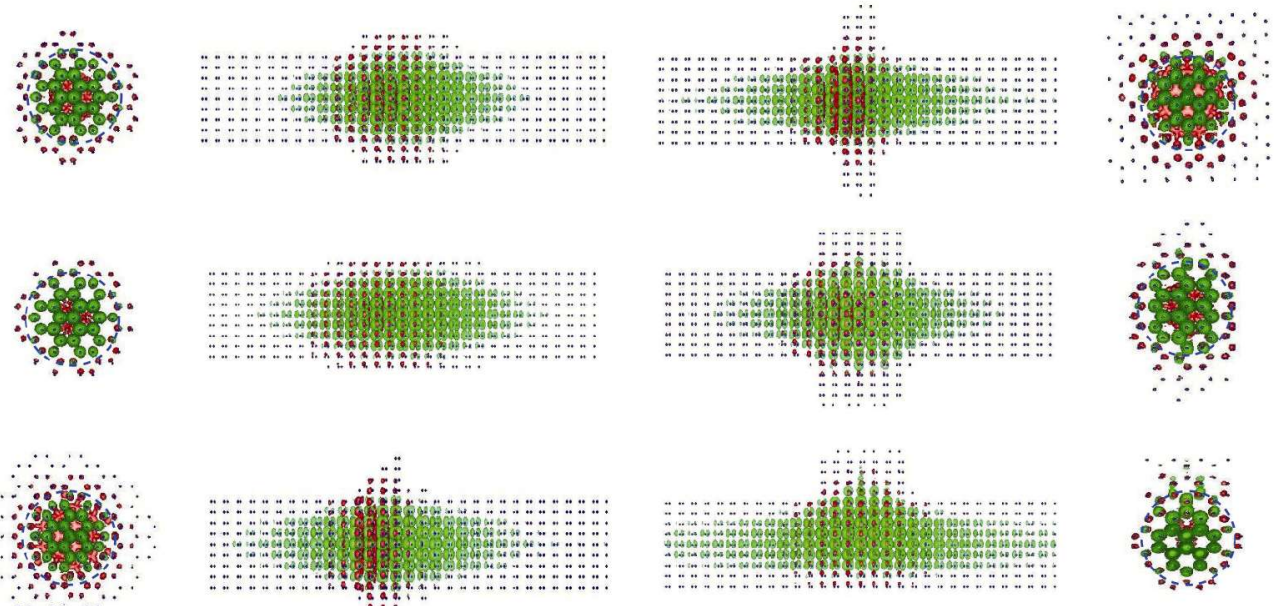

(6)

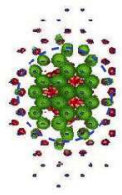

(5)

(7)

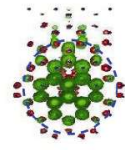

(8)

Figure 1. The isosurface plots of the squares of the wavefunctions. Both the top views and the side views of the bulged nanowires are shown. The small blue dots denote the $\mathrm{Cd}$ and $\mathrm{Se}$ atoms. The green isosurfaces are for the VBM states while the red isosurfaces are for the CBM states. The isosurface values for both the VBM and CBM are 0.0002 (electron/Bohr $\left.{ }^{3}\right)$. The dashed line circles in the top views denote the uniform nanowire. The index (1) to (8) denote wire_1 to wire_8. 

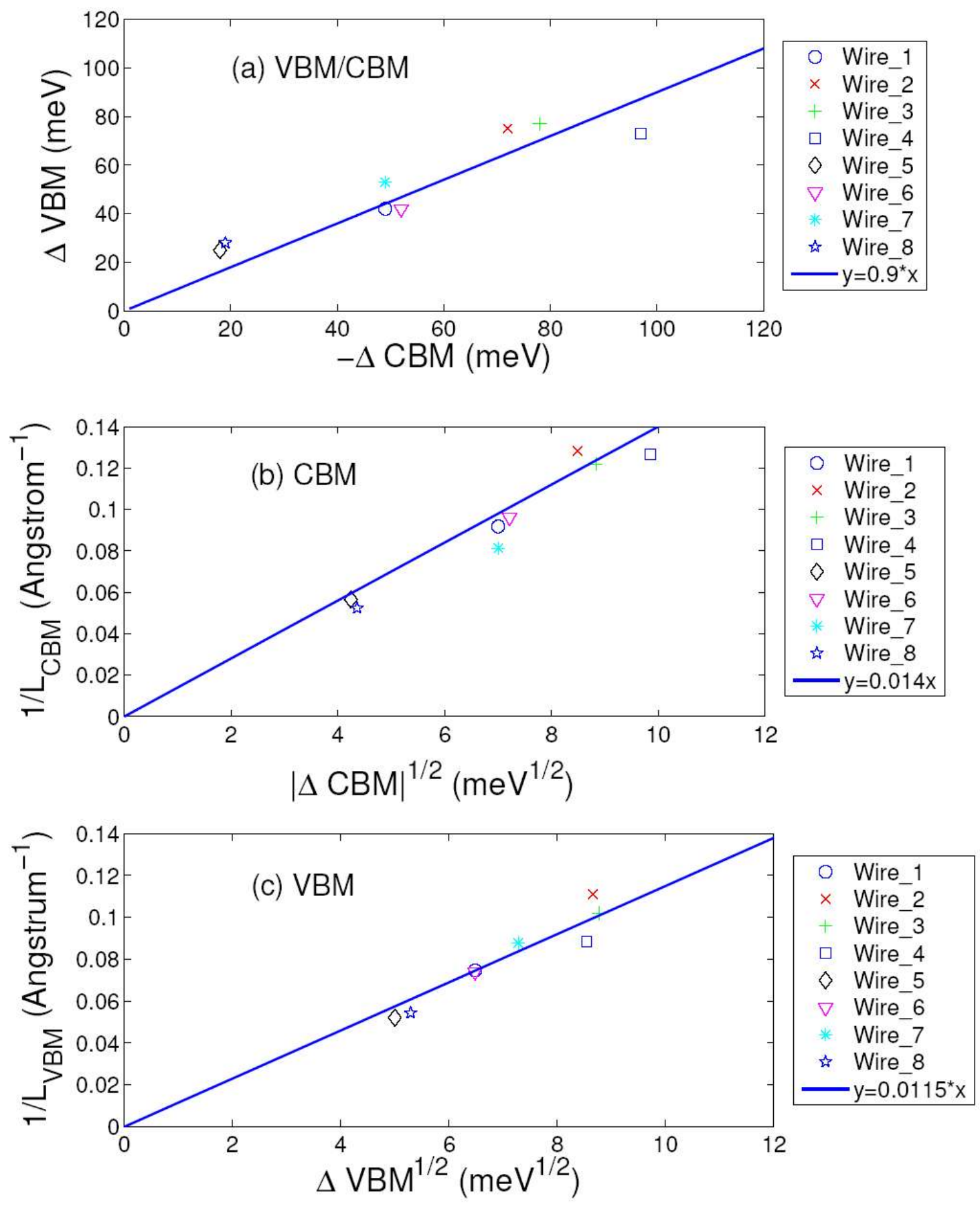

Figure 2. The correlations between VBM binding energy and CBM binding energy (a); and the relationship between the decay length $\mathrm{L}$ and the binding energy for CBM (b); and VBM (c). The lines are used to represent the overall trends. 


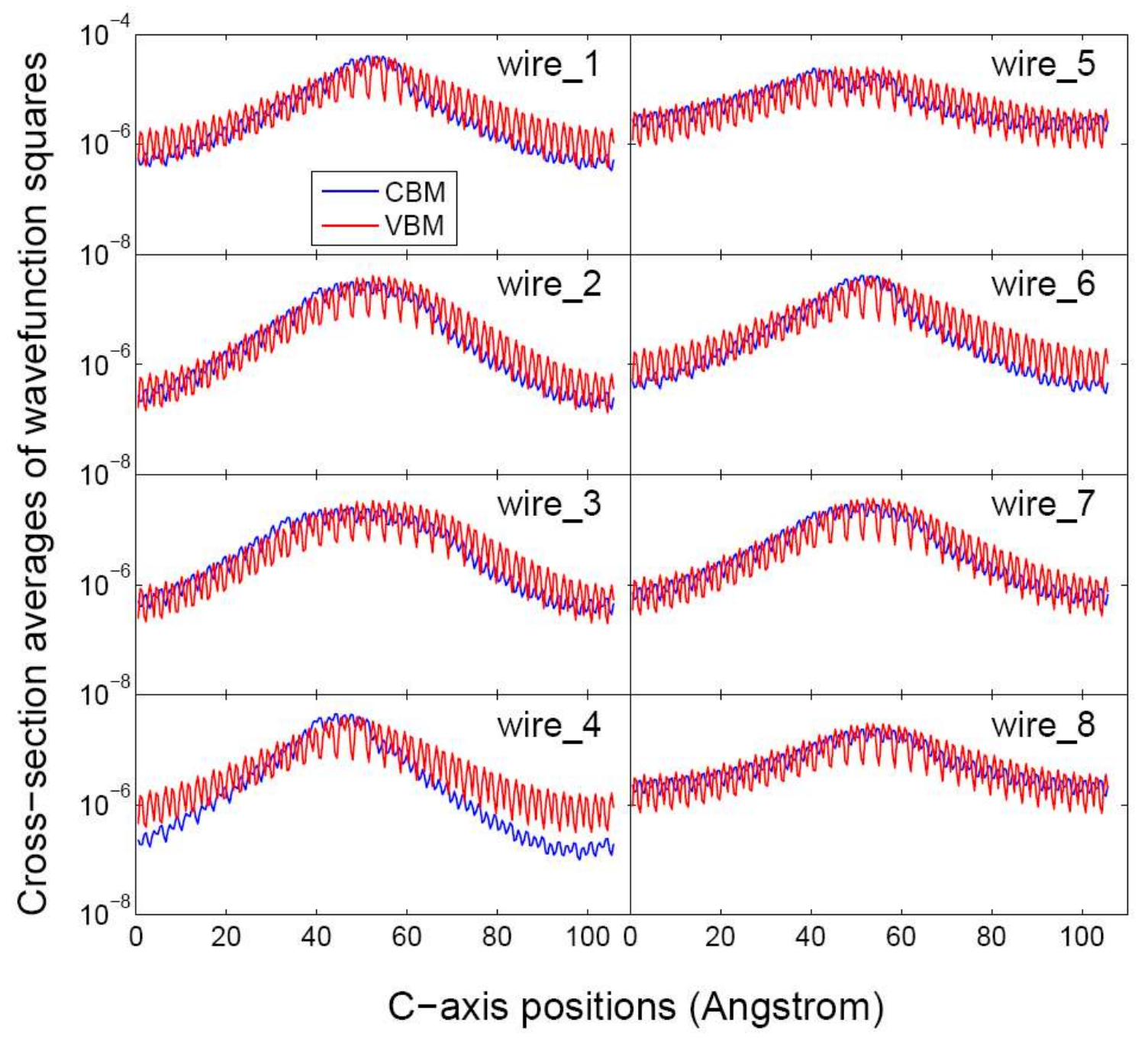

Figure 3. The cross section averaged wavefunction squares for the CBM and VBM states. One wurtzite unit cell length $(7 \AA)$ in the c-axis direction corresponds to 32 numerical grid points. The shapes of the wire_1 to wire_ 8 are described in Table I and illustrated in Fig.1. 


\section{References}

1. J. Hu, L. Li, W. Yang, L. Manna, L.W. Wang and A.P. Alivisatos, Science 292, 2060 (2001).

2. J. Li and L.W. Wang, Nano Lett. 3, 1357 (2003).

3. M. Law, J. Goldberg and P. Yang, Annu. Rev. Mater. Res. 34, 83 (2004).

4. T.J. Trentler, K.M. Hickman, S.C. Goel, A.M. Viano, P.C. Gibbons and W.E. Buhro, Science 270, 1791 (1995).

5. H. Yu, J. Li, R.A. Loomis, P.C. Gibbons, L.W. Wang and W.E. Buhro, J. Am. Chem. Soc. 125, 16168 (2003).

6. Y. Wang and S.J. Sibener, J. Phys. Chem. B 106, 12856 (2002).

7. D. Xu, X. Shi, G. Guo, L. Gui and Y. Tang, J. Phys. Chem. B 104, 5061 (2000).

8. K.S. Cho, D.V. Talapin, W. Gaschler and C.B. Murray, J. Am. Chem. Soc. 127,7140 (2005).

9. Z. Tang, N.A. Kotov and M. Giersig, Science 297, 237 (2002).

10. X. Duan, C.M. Lieber and Adv. Mater. 4, 298 (2000).

11. The compactness of a cross section can be measured from the average distance of the atomic positions to the center of mass point. Smaller this average, larger the compactness. Thus, for a given area, the sphere is the most compact shape.

12. L.W. Wang and A. Zunger, Phys. Rev. B 53, 9579 (1996).

13. L.W. Wang and A. Zunger, Phys. Rev. B 51, 17398 (1995).

14. L.W. Wang and A. Zunger, J. Chem. Phys. 100, 2394 (1994). 
15. J. Hu, L. Li, L.W. Wang and A.P. Alivisatos, J. Phys. Chem. 106, 2447 (2002). 